\title{
Assistência de enfermagem diante da segurança do paciente idoso
}

Nursing care in the light of elderly patient safety

Atención de enfermería a la luz de la seguridad del paciente anciano

Anna Carolina Lomelino Lemos Medeiros ORCID: https://orcid.org/0000-0002-8835-9389 Universidade de Vassouras, Brasil E-mail: carolinalomelino@outlook.com

Andréa Capistrano das Neves Cunha ORCID: https://orcid.org/0000-0002-8396-1020 Faculdade Bezerra de Araújo, Brasil E-mail: andcapis@hotmail.com

Débora Maria da Silva Xavier ORCID: https://orcid.org/0000-0002-1381-544X

Universidade Federal de Pernambuco, Brasil E-mail: debxavier@hotmail.com

Eliane de Lira Goulart Caminha ORCID: https://orcid.org/0000-0002-9061-2494

Universidade Veiga de Almeida, Brasil

E-mail: elianegoulart1984@gmail.com

Fábio Angelo de Medeiros

ORCID: https://orcid.org/0000-0002-2077-1457

Universidade Estácio de Sá, Brasil

E-mail: fabio.angelo36@gmail.com

Kenia Oliveira Barbosa da Hora ORCID: https://orcid.org/0000-0002-2887-7408 Universidade Federal do Rio de Janeiro, Brasil E-mail: kenia.0.barbosa@ gmail.com

Larissa Carvalho Teixeira ORCID: https://orcid.org/0000-0002-4439-7578 Universidade Estácio de Sá, Brasil

E-mail: larissacarvalho.tjc@gmail.com

Larissa Nunes dos Santos Sales ORCID: https://orcid.org/0000-0002-4207-0870 Faculdade UNIABEU, Brasil

E-mail: larissanunesenf@hotmail.com Luciane Motta Baltar

ORCID: https://orcid.org/0000-0003-4571-5459

Universidade Estácio de Sá, Brasil E-mail: lumbaltar@gmail.com

Luiza Pacífico Cardoso

ORCID: https://orcid.org/0000-0002-8547-2466 Universidade de Brasília, Brasil E-mail: luiza.cardoso64@gmail.com

Marcus Vinícius Braga Esteves ORCID: https://orcid.org/0000-0001-5450-1693

Universidade Estácio de Sá, Brasil E-mail:estevesbmarcus@outlook.com

Milena Moraes Santos da Silva ORCID: https://orcid.org/0000-0001-5461-8576

Universidade Estácio de Sá, Brasil

E-mail: milenamoraes010299@gmail.com

Priscilla Duarte Soares Correa ORCID:https://orcid.org/0000-0002-9227-1259

Universidade Estácio de Sá, Brasil e-mail:priscillakdsoares@yahoo.com.br

Priscila de Almeida Cunha Silva ORCID: https://orcid.org/0000-0002-2322-634X

Universidade Estácio de Sá, Brasil

E-mail: priscilaalmeida_c@yahoo.com.br Raiane Monteiro Rodrigues da Silva ORCID: https://orcid.org/0000-0002-1717-7254

Universidade Iguaçu, Brasil

E-mail: raiimonteiroo@gmail.com 


\author{
Ronilce Rozendo Amorim \\ ORCID: https://orcid.org/0000-0003-22916055 \\ Universidade Estácio de Sá, Brasil \\ E-mail: ronilceamorym@gmail.com \\ Valéria Cristina Brandão Marinho \\ ORCID: https://orcid.org/0000-0002-9344-6161 \\ Universidade Estácio de Sá, Brasil \\ E-mail: vcbmarinho@gmail.com \\ Vítor Diego de Pontes Simões \\ ORCID: https://orcid.org/0000-0002-9317-4549 \\ Centro Universitário Augusto Motta, Brasil \\ E-mail: simoes872004@yahoo.com.br \\ Mariana Lopes Teixeira \\ ORCID: https://orcid.org/0000-0002-1382-3271 \\ Universidade Estácio de Sá, Brasil \\ E-mail: marianalopestx@gmail.com \\ Larissa Lessa dos Santos \\ ORCID: https://orcid.org/0000-0002-8947-6691 \\ Universidade Estácio de Sá, Brasil \\ E-mail: larissalessaa@hotmail.com
}

\title{
Resumo
}

Introdução: A segurança do paciente foi classificada como uma atribuição de enfermagem pela Organização Mundial de Saúde com intuito de reduzir os danos desnecessários à saúde e proporcionar eficácia durante as internações. Objetivo: Descrever a importância da assistência de enfermagem relacionada a segurança do paciente idoso. Metodologia: Trata-se de uma revisão integrativa, onde os documentos para a pesquisa foram obtidos e escolhidos estudos por meio de coleta de dados de publicações de autores de referência na área com posterior leitura crítica dos títulos e resumos, na Biblioteca Virtual em Saúde (BVS), utilizando os seguintes Descritores em Ciência da Saúde (DESC) para a busca e seleção de dados: "Enfermeiro", "Segurança do paciente" e "Idoso", utilizando o operador booleano "AND" entre eles na pesquisa. Resultados: Foi elaborada uma categoria para discutir sobre a temática, sendo o título "As principais medidas de segurança do paciente idoso utilizadas pelo enfermeiro". Considerações finais: É essencial que a assistência clínica ao ser humano inclua um espaço seguro, no qual o cliente seja elucidado sobre a terapêutica, restrições, ameaças e precauções necessárias no decorrer do tratamento. Nesta circunstância, a enfermagem como saber e ocupação que assiste indivíduos e grupos de forma ímpar e multidimensional precisa que conceitos, a estudos e prática estejam ligados para promover um tratamento clínico de confiança.

Palavras-chave: Enfermeiro; Segurança do paciente; Idoso.

\begin{abstract}
Introduction: Patient safety was classified as a nursing assignment by the World Health Organization with the aim of reducing unnecessary harm to health and providing efficacy during hospitalizations. Objective: To describe the importance of nursing care related to the safety of elderly patients. Methodology: This is an integrative review, where documents for the research were obtained and studies were chosen through data collection from publications by reference authors in the area, with subsequent critical reading of titles and abstracts, in the Virtual Health Library ( BVS), using the following Health Science Descriptors (DESC) for the search and selection of data: "Nurse", "Patient safety" and "Elderly", using the Boolean operator "AND" among them in the research. Results: A category was created to discuss the theme, entitled "The main safety measures used by nurses for elderly patients". Final considerations: It is essential that clinical care for human beings includes a safe space, in which the client is informed about the therapy, restrictions, threats and necessary precautions during the treatment. In this circumstance, nursing as a knowledge and occupation that assists individuals and groups in a unique and multidimensional way needs that concepts, studies and practice are linked to promote reliable clinical treatment.
\end{abstract}

Keywords: Nurse; Patient safety; Old man.

\section{Resumen}

Introducción: La seguridad del paciente fue catalogada como una asignación de enfermería por la Organización Mundial de la Salud con el objetivo de reducir los daños innecesarios a la salud y brindar eficacia durante las hospitalizaciones. Objetivo: Describir la importancia de los cuidados de enfermería relacionados con la seguridad del paciente anciano. Metodología: Se trata de una revisión integradora, donde se obtuvieron los documentos para la investigación y se seleccionaron los estudios mediante recolección de datos de publicaciones de autores de referencia en el área, con posterior lectura crítica de títulos y resúmenes, en la Biblioteca Virtual en Salud (BVS), utilizando los siguientes Descriptores de Ciencias de la Salud (DESC) para la búsqueda y selección de datos: "Enfermera", "Seguridad del paciente" y "Anciano", utilizando el operador booleano "Y" entre ellos en la investigación. Resultados: Se creó una categoría para discutir el tema, titulada "Las principales medidas de seguridad que utilizan las enfermeras para el paciente anciano". Consideraciones finales: Es fundamental que la atención clínica al ser humano 
incluya un espacio seguro, en el que se informe al cliente sobre la terapia, restricciones, amenazas y precauciones necesarias durante el tratamiento. En esta circunstancia, la enfermería como conocimiento y ocupación que asiste a individuos y grupos de manera única y multidimensional necesita que los conceptos, los estudios y la práctica estén vinculados para promover un tratamiento clínico confiable.

Palabras clave: Enfermera; Seguridad del paciente; Anciano.

\section{Introdução}

A segurança do paciente foi classificada como uma atribuição de enfermagem pela Organização Mundial de Saúde (OMS) com intuito de reduzir os danos desnecessários à saúde e proporcionar eficácia durante as internações para a realização dos cuidados mínimos aceitáveis para uma assistência de qualidade. (Silva et al., 2016).

O enfermeiro é o profissional responsável por assegurar uma assistência segura aos pacientes, família e sociedade. É necessário orientar a equipe sobre os atos iatrogênicos que possam ocorrer no setor atuante, riscos e intercorrências durante as práticas de enfermagem. (COFEN, 2015).

Para ter a implementação de medidas que previnam acidentes com os pacientes internados, como: protocolos e atividades padronizadas pela empresa, a gestão do serviço precisa ser ativa, participativa, estimular o bom funcionamento da equipe para melhor organização do hospital, evitando aspectos problemáticos na assistência e possíveis adversidades que possam surgir. (Silva et al., 2016).

As principais metas de qualquer setor de internação, principalmente de idosos são: identificação de pacientes para reduzir incidentes, prevenção de quedas, higienização das mãos, segurança medicamentosa quanto a prescrição médica e administração de fármacos e prevenção de lesão por pressão. (Reis et al., 2012).

A relevância para o público da enfermagem se faz com o objetivo de informar e propor uma reflexão sobre o assunto, uma vez que será de grande valia esse conhecimento para a assistência.

A pesquisa possui o objetivo de descrever a importância da assistência de enfermagem relacionada a segurança do paciente idoso.

\section{Metodologia}

Este estudo trata-se de uma revisão integrativa de literatura, de abordagem qualitativa, o que proporciona uma síntese do conhecimento e a incorporação da aplicabilidade de resultados de estudos significativos na prática (Souza, Silva, \& Carvalho, 2010)

A pesquisa de abordagem qualitativa é um método de estudo que busca descrever e explicar os fenômenos pesquisados, através de estudos observacionais e entrevistas contextuais. Devendo esta metodologia ser estruturada a modo de responder à questão da pesquisa formulada, com uma análise lógica dos dados levantados, para fomentar uma conclusão coerente, sendo seu objetivo principal a observância dos fenômenos apresentados. (Cyriaco et al., 2017)

O estudo qualitativo não se preocupa com o aprofundamento da compreensão de um grupo social, de uma organização, se opondo ao pressuposto que defende um modelo único de pesquisa para todas as ciências, já que as ciências sociais têm sua especificidade pressupondo uma metodologia própria. Goldenberg (1997).

Bardin (2016) define que a pesquisa qualitativa é a que se fundamenta principalmente em análises qualitativas, caracterizando-se, em princípio, pela não utilização de instrumental estatístico na análise dos dados.

A proposta de construção de revisão integrativa de acordo com Mendes et al., (2008) é um método com etapas distintas a serem seguidas. De acordo com esses critérios, esta revisão deve conter seis etapas, sendo elas: Estabelecimento da hipótese ou questão de pesquisa; Amostragem ou busca na literatura; Categorização dos estudos; avaliação dos estudos incluídos na revisão; Interpretação dos resultados; Síntese do conhecimento ou apresentação da revisão. 
Para a construção e elaboração da questão norteadora utilizou o método voltado para a pesquisa clínica. A estratégia PICo possui um acrônimo para paciente, intervenção e contexto. Sendo elementos fundamentais na formulação da questão norteadora para assim ser realizada a busca bibliográfica. (Santos et al., 2007).

Quadro 1: Estratégia PICo, para definição da questão norteadora.

\begin{tabular}{|l|l|l|}
\hline Acrônimo & Descrição & Componentes da questão \\
\hline P & Participantes & Idosos \\
\hline I & Intervenção & Segurança do paciente \\
\hline Co & Contexto & Assistência de enfermagem relacionada a segurança do paciente idoso \\
\hline
\end{tabular}

Fonte: Autores (2021).

O processo de interpretação dos dados é considerado a fase mais complexa, sendo necessário a iniciação do estudo com um entendimento e incorporação de definições filosóficas e epidemiológicas que estruturam a pesquisa, desde o processo de seleção do objeto de estudo da pesquisa. (Minayo, 2011)

Os documentos foram obtidos e escolhidos estudos por meio de coleta de dados de publicações de autores de referência na área com posterior leitura crítica dos títulos e resumos, na Biblioteca Virtual em Saúde (BVS), utilizando os seguintes Descritores em Ciência da Saúde (DESC) para a busca e seleção de dados: "Enfermeiro", "Segurança do paciente" e "Idoso", utilizando o operador booleano "AND" entre eles na pesquisa.

Foram definidos os seguintes critérios de inclusão: artigos indexados na íntegra e disponíveis para leitura, em Língua Portuguesa e texto completo, com recorte temporal de 10 anos (2012 a 2021). Como critérios de exclusão foram descartados os artigos em repetição ou que não estavam em concordância com a temática proposta. Após empregar os critérios de inclusão e exclusão na busca bibliográfica, foi possível observar uma imensa escassez de publicações e estudos referentes a temática, emergindo um total de 4 artigos para compor este trabalho, alinhados ao tema proposto.

\section{Resultados e Discussão}

Os resultados apresentados no estudo foram descritos no Quadro 1 com elementos que compõem os estudos analisados. 
Quadro 2: Características dos artigos analisados no período de 2016 a 2021.

\begin{tabular}{|c|c|c|c|c|c|}
\hline Título & Autoria & Ano & Objetivo & Métodos & Considerações Finais \\
\hline $\begin{array}{l}\text { Teoria do conforto } \\
\text { como subsídio para o } \\
\text { cuidado clínico de } \\
\text { enfermagem }\end{array}$ & $\begin{array}{l}\text { Raquel Silveira Mendes; } \\
\text { Amanda Miranda Cruz; } \\
\text { Dafne Paiva Rodrigues; } \\
\text { Julianara } \\
\text { Figueiredo; } \\
\text { Ana Virgínia de Melo }\end{array}$ & 2016 & $\begin{array}{l}\text { Refletir sobre a teoria do } \\
\text { conforto e reus } \\
\text { fundamentos teórico- } \\
\text { filisóficos como subsídio } \\
\text { para o cuidado clínico de } \\
\text { enfermagem ao indivíduo, } \\
\text { família e comunidades. }\end{array}$ & $\begin{array}{l}\text { Estudo } \\
\text { qualitativo }\end{array}$ & $\begin{array}{l}\text { Permite a compreensão das } \\
\text { necessidades do outro e } \\
\text { possibilita que o cuidado } \\
\text { seja realizado de forma } \\
\text { individualizada. }\end{array}$ \\
\hline $\begin{array}{l}\text { Risco de lesão por } \\
\text { pressão em idosos } \\
\text { com } \\
\text { comprometimento na } \\
\text { realização de } \\
\text { atividades diárias }\end{array}$ & $\begin{array}{l}\text { Vanete Aparecida de } \\
\text { Souza Vieira; } \\
\text { Mariana Diniz Costa } \\
\text { Santos; } \\
\text { Amanda do Nascimento } \\
\text { Almeida; } \\
\text { Cristiane Chaves de } \\
\text { Souza; } \\
\text { Mariana Ferreira Vaz } \\
\text { Gontijo Bernardes; } \\
\text { Luciana Regina Ferreira } \\
\text { da Mata }\end{array}$ & 2018 & $\begin{array}{l}\text { Avaliar o risco de } \\
\text { institucionalizados com } \\
\text { comprometimentos na } \\
\text { realização das atividades } \\
\text { de vida diárias } \\
\text { desenvolverem lesão por } \\
\text { pressão. }\end{array}$ & $\begin{array}{l}\text { Estudo } \\
\text { transversal } \\
\text { qualitativo }\end{array}$ & $\begin{array}{l}\text { O uso de escalas preditivas } \\
\text { como as de Braden e Katz } \\
\text { proporciona parâmetros } \\
\text { para o enfermeiro planejar } \\
\text { cuidados com a pele de } \\
\text { modo individualizado, } \\
\text { visando a segurança e bem- } \\
\text { estar dos idosos } \\
\text { institucionalizados. }\end{array}$ \\
\hline $\begin{array}{l}\text { Quedas em pacientes } \\
\text { cirúrgicos: Subsídios } \\
\text { para o cuidado de } \\
\text { enfermagem seguro }\end{array}$ & $\begin{array}{l}\text { Marco Antonio de Goes } \\
\text { Victor; } \\
\text { Melissa de Freitas Luzia; } \\
\text { Isis Marques Severo; } \\
\text { Miriam de Abreu } \\
\text { Almeida; } \\
\text { Marta Georgina Oliveira } \\
\text { de Goes; } \\
\text { Amália de Fátima } \\
\text { Lucena }\end{array}$ & 2017 & $\begin{array}{lcr}\text { Descrever } & \text { o } & \text { evento } \\
\text { adverso } & \text { queda } & \text { em } \\
\text { pacientes internados } & \mathrm{em} \\
\text { unidades cirúrgicas } & \end{array}$ & $\begin{array}{l}\text { Estudo } \\
\text { retrospectivo }\end{array}$ & $\begin{array}{l}\text { A queda é um evento } \\
\text { multicausal e o enfermeiro } \\
\text { deve estar atendo aos seus } \\
\text { fatores de risco para } \\
\text { intervir e prevenir o } \\
\text { mesmo. }\end{array}$ \\
\hline $\begin{array}{l}\text { Assistência re de } \\
\text { enfermagem segura } \\
\text { e qualificada: } \\
\text { Avaliação do risco } \\
\text { cirúrgico no cuidado } \\
\text { perioperatório ao } \\
\text { idoso }\end{array}$ & $\begin{array}{l}\text { Melissa Orlandi Honório } \\
\text { Locks; } \\
\text { Darla Lusia Ropelato } \\
\text { Fernandez; } \\
\text { Lúcia Nazareth Amante; } \\
\text { Karina Silveira de } \\
\text { Almeida } \\
\text { Hammerschmidt; } \\
\text { Luciara Fabiane Sebold; } \\
\text { Juliana Balbinot Reis } \\
\text { Girondi }\end{array}$ & 2016 & $\begin{array}{l}\text { Discutir o uso de escalas } \\
\text { para avaliar o risco } \\
\text { cirúrgico de idosos no } \\
\text { período perioperatório. }\end{array}$ & $\begin{array}{l}\text { Comentário } \\
\text { crítico }\end{array}$ & $\begin{array}{l}\text { As escalas para avaliação } \\
\text { de risco cirúrgico } \\
\text { apresentadas podem ser } \\
\text { incorporadas à prática } \\
\text { cotidiana dos enfermeiros, } \\
\text { mediante o estabelecimento } \\
\text { de plano de cuidados } \\
\text { individualizado. }\end{array}$ \\
\hline
\end{tabular}

Fonte: Autores (2021).

Após análise dos artigos selecionados neste estudo foi construída a seguinte categoria.

\section{Categoria: Medidas de segurança do paciente idoso utilizadas pelo enfermeiro.}

A assistência clínica realizada pela enfermagem são intervenções profissionais baseadas em experiência científica, focadas no restabelecimento da saúde objetivando a independência do indivíduo. A atenção da equipe, especialmente a atenção clínica, precisa ser desenvolvida e ir mais adiante do que está perceptível, levando em conta o ser humano em si e suas experiências. (Mendes et al., 2016).

Assistência resolutiva e eficiente é ofertada ao cliente, garantindo proteção graças ao entendimento técnico e científico da equioe de enfermagem e compromisso com as práticas sistêmicas, de uma maneira que tente diminuir consequências indesejadas e acautelar possíveis complicações. (Locks et al., 2016).

Para a enfermagem, assistir do outro significa responder às suas carências de maneira sensível, prestativa e solidária através de ações de cuidado realizadas para proporcionar o refrigério e o aconchego. Ao atender, a equipe de enfermagem promove a segurança do cliente por meio da execução de práticas que colaboram para a melhoria do bem-estar, nas diversas circunstâncias, sendo elas: física, psicológica, religiosa, social e cultural. (Mendes et al., 2016).

É essencial que o enfermeiro atue com a intenção de prevenir dano ao cliente, uma dessas medidas é evitar o aparecimento das lesões por pressão, tendo em vista que o surgimento destas pode reduzir a independência do cliente idoso, 
além disso, o submeter a complicações oriundas do comprometimento da plenitude do tecido. Para isso, o trabalhador pode usar artifícios como ferramentas padronizadas que proporcionem reconhecer indivíduos em perigo de evoluir para lesão por pressão e, desta forma, aperfeiçoar práticas preventivas, cooperando para o cuidado da condição de saúde dos sêniores. (Vieira et al., 2018).

O reconhecimento dos usuários, das eventualidades adversas associados à medicações e às intervenções cirúrgicas, aparecimento de lesões por pressão, eventos de segurança, infecções e diálogo no espaço de trabalho de saúde são fundamentais para fortalecer atividades de assistência com mais qualidade e menor risco de danos. (Mendes et al., 2016).

O crescimento da ancianidade e da nulidade eleva o predomínio de lesão por pressão (LP) e os problemas e agravos resultantes desta. É válido enfatizar que a cautela com o surgimento de LP é uma das seis metas preconizadas pelo Programa Nacional de Segurança do Paciente, que compreende boas condutas, diminuição de falhas e inexistência de danos evitáveis a um usuário no momento do processo de assistência de saúde. (Vieira et al., 2018).

Nota-se a importância de efetuar ações de prevenção como elevação de cabeceira de cama em até $30^{\circ}$, utilização de colchões específicos para distribuir pressão, usar lençol móvel para mobilizar ou mover clientes com mobilidade restrita, modificações de posicionamento, efetuação de fisioterapia motora e trocas frequentes de fraldas. Essas atitudes são de compromisso dos profissionais de enfermagem e representam-se como imprescindíveis para assegurar o cliente contra lesões por pressão. (Vieira et al., 2018).

Outro ponto importante são as práticas cirúrgicas em indivíduos idosos, que estão se apresentando cada vez mais comuns devido à longevidade da sociedade e elevação da produção de doenças de caráter cirúrgico. Desta forma, agravamentos agudos e problemas crônicos relacionados às mudanças associados ao envelhecimento são condições primordias a serem analisadas na fase perioperatória. (Locks et al., 2016).

O estado pós-operatório também se destaca como propício para queda, por conta de obstáculos para deambulação e mobilidade, a utilização de medicamentos sedativos e analgésicos, o uso de equipamentos de auxílio à movimentação, existência de cateteres e drenos, além de possibilitar um quadro de hipotensão postural, por instabilidade de volume do sangue, no momento ou depois do ato cirúrgico. (Victor et al., 2017).

$\mathrm{O}$ âmbito cirúrgico e os perigos relacionados à operação são contextos que configuram a necessidade de estruturação correta para certificar a proteção no cuidado ao cliente. A enfermagem exerce uma função importantíssima nesse quesito, visto que a assistência de enfermagem pode ajudar em uma melhor fase perioperatória, fase com redução de agravos e maior recuperação. (Locks et al., 2016).

A existência de complicações cirúrgicas está diretamente ligada à idade, histórico de comorbidades e cirurgias de emergência. Por tanto, os idosos estão mais suceptíveis à morbimortalidade, o que requer maior alerta da equipe de saúde. (Locks et al., 2016).

Para beneficiar o progresso destes, torna-se necessário que a tríade estudos, teoria e ações sigam juntos. Associação da Teoria do Conforto e a segurança do paciente destinam-se a assegurar a Enfermagem como profissão da área da saúde e ao mesmo tempo em norma científica. (Mendes et al., 2016).

\section{Considerações Finais}

É essencial que a assistência clínica ao ser humano inclua um espaço seguro, no qual o cliente seja elucidado sobre a terapêutica, restrições, ameaças e precauções necessárias no decorrer do tratamento. Nesta circunstância, a enfermagem como ciência que assiste indivíduos e grupos de forma ímpar e multidimensional precisa que conceitos, a estudos e prática estejam ligados para promover um tratamento clínico de confiança. 
Muitas transformações positivas têm acontecido nos últimos anos para aprimorar a segurança do paciente, produzindo-se diversos projetos e ações que estão concedendo maior segurança ao usuário. Está, também, em crescimento, a produção de hábitos voltados para a segurança possibilitando os estabelecimentos de saúde e aos trabalhadores desfrutarem de componentes e instrumentos em benefício de um cuidado de qualidade.

A segurança do paciente tem sido considerada como um constituinte primordial de qualidade das atividades de saúde mundialmente. Portanto, para garantir a segurança do paciente idoso, o profissional enfermeiro é parte fundamental do processo, pois a mesma é condição crucial no gerenciamento de qualidade a qual está associada à assistência elaborada nos institutos de saúde pelo enfermeiro e sua equipe.

Espera-se que as pesquisas a respeito do tema "segurança do paciente idoso" sejam capazes de colaborar com a compreensão e desenvolvimento dos trabalhadores de enfermagem e contar como alerta para a implementação de práticas que possibilitem um cuidado eficiente e sem prejuízos para a sociedade.

\section{Referências}

Bardin, L. (2016). Análise de conteúdo. Editora Almedina.

Cyriaco, A. F., Nunn, D., Amorim, R. F., Falcão, D. P., \& Moreno, H. (2017). Pesquisa Qualitativa: conceitos-chave e um breve panorama de sua aplicação em geriatria/gerontologia. Envelhecimento, 11(1), 4-9.

Conselho Federal De Enfermagem (COFEN). Resolução n ${ }^{o}$ 311/07. Aprova a reformulação do Código de Ética dos Profissionais de Enfermagem. In: Conselho Regional De Enfermagem De Minas Gerais (COREN-MG). Legislação e normas. 14(1), 37-54, 2015.

Goldenberg, M. (1997). A arte de pesquisar. Record.

Locks, M. O; Fernandez, D. L., Amante, L. N., Hammerschimidt, K. S., Sebold, L. F., \& Girondi, J. B. (2016). Safe and effective nursing care: assessment of surgical risks in perioperative care of elderly patients. Cogitare Enferm, 21(3):01-07.

Mendes, K., Silveira, R. C., \& Galvão, C. M. (2008). Revisão integrativa: método de pesquisa para a incorporação de evidências na saúde e na enfermagem. Texto Contexto Enfermagem, 17(4): 758-64.

Mendes, R. S; Cruz, A. M; Figueiredo, J. V; Rodrigues, D. P., \& Melo, A. V. (2016). Teoria do conforto como subsídio para o cuidado clínico de enfermagem. Cienc Cuid Saude, 15(2):390-395.

Minayo, M. C. (2011). Pesquisa social. Teoria, método e criatividade. Editora Vozes.

Reis, C.T., Martins, M., \& L. J. (2012). A segurança do paciente como dimensão da qualidade do cuidado de saúde - um olhar sobre a literatura. Ciência \& Saúde Coletiva, 2029-2036.

Santos, C. M., Pimenta, C. B., \& Nobre, M. R. (2007). The PICO Strategy for there search question construction and evidence search. Ver. Latino-Am. Enfermagem, 15(3), 508-511.

Silva, A. T., Alves, M. G., Sanches, R. S., Terra, F. S., \& Resck, Z. M. (2016). Assistência de enfermagem e o enfoque da segurança do paciente no cenário brasileiro. Saúde debate, 11(40), 292-301.

Souza, G. R. M., Cazola, L. H. de O., \& Pícoli, R. P. (2018). Atuação do enfermeiro da atenção primária à saúde na assistência oncológica: revisão integrativa. Cogitare Enfermagem, 23(4), e58152. Epub 31 de janeiro de 2018.

Souza, M. T., Silva, M. D., \& Carvalho, R. (2010). Revisão integrativa: o que é e como fazer. Einstein, 8(1), $102-106$.

Victor, M. A., Luzia, M. F., Severo, I. M., Almeida, M. A., Goes, M. G. \& Lucena, A. F. Quedas em pacientes cirúgicos: Subsídio para o cuidado de enfermagem seguro.

Vieira V. A. S., Santos, M. D. C., Almeida, N. A., et al. Risco de lesão por pressão em idosos com comprometimento na realização de atividades diárias. Revista de Enfermagem do Centro-Oeste Mineiro. 2018;8:e2599. 